\title{
A REDUCTION OF THE POINCARE CONJECTURE TO OTHER CONJECTURES. II
}

\author{
BY C. D. PAPAKYRIAKOPOULOS ${ }^{1}$
}

Communicated by Lipman Bers, January 29, 1963

In a recent note I formulated two conjectures [2, p. 365], which imply the Poincaré conjecture. Bernard Maskit produced a counter example to the second of those conjectures [1]. In the present note I modify those conjectures, in a way suggested by his counter example and his two examples. The modified conjectures imply the Poincare conjecture and avoid the obstacles raised by his examples. To avoid confusion, in the remainder of this note, I will be referring to the counter example of Maskit as the first example of [1] and to the other ones as the second and third examples of [1] respectively.

In this note we use the notations and conventions of [2]. In the first example of [1] the word $b_{1}\left[b_{1}^{-1}, b_{2}\right]$ is not cyclically reduced but, as an element of $F$, is representable by a simple loop on $N$. In the second example of [1] the word $b_{1}\left[b_{2}^{-1}, a_{1}^{-1}\right]$ is cyclically reduced but, as an element of $F$, is not representable by a simple loop on $N$. This can be seen by using the algorithm of Bruce L. Reinhart $[4, \S 3]$. In the third example of [1] the word $b_{1}\left[b_{2}, a_{2}\right]$ is cyclically reduced and moreover, as an element of $F$, is representable by a simple loop on $N$.

Before we express the modified conjectures, let us compare the first and third examples of [1]. In the first example $A_{1}^{\prime}$, see $[2$, p. 365, 11. 3-9], is obtained through a transformation of $B_{1}$ by $B_{2}$. In the third example we first have to change our "coordinate system" on $N$, and consider a new system $A_{i}^{\prime \prime}, B_{i}^{\prime \prime}, i=1,2$, such that $B_{1}^{\prime \prime}$ or $B_{2}^{\prime \prime}$ represents the element $b_{1}\left[b_{2}, a_{2}\right]$ or $b_{2}\left[b_{1}, a_{1}\right]$ of $F$. We then observe that $A_{i}$ is obtained through a transformation of $A_{i}^{\prime \prime}$. Thus the first and third examples of [1] have a common feature. Namely, the first is obtained by transforming $B_{1}$, while the third is obtained by transforming $A_{1}$ (and changing the coordinate system). This common feature suggests that, we have to "pass to the geodesics," before expressing the conjectures.

Let $N$ be an orientable closed surface of genus $p \geqq 2$, and let $A_{i}, B_{i}, i=1, \cdots, p$, be a fundamental system of $N$ with base point $o$. As is well known, $N$ has certain hyperbolic metrics, imposed on it by Poincaré, and therefore there are geodesics on $N$, see [3, No. 3].

Let now $G_{i}$ and $H_{i}$ be the primary closed geodesics on $N$, cor-

1 The author is an Alfred P. Sloan Foundation Research Fellow. 
responding to $A_{i}$ and $B_{i}$ respectively, $i=1, \cdots, p[3$, Lemma (12.2)]. Then $G_{i}$ and $H_{i}$ have only one point $q_{i}$ in common [3, Nos. 3 and 12], any two of the pairs $G_{i}, H_{i}$ different from each other are disjoint, and if we cut $N$ along the $G$ and $H$ 's we obtain a 2-sphere with $p$ holes having boundaries $G_{i} H_{i} G_{i}^{-1} H_{i}^{-1}$.

Let $G_{i}^{\prime}, i=1, \cdots, p$, be primary simple closed geodesics on $N$, any two different ones being disjoint, and such that $G_{i}^{\prime}$ is homologous to $H_{i}$ on $N$. Let $o_{i}$ be some common point of $G_{i}$ and $G_{i}^{\prime}$.

Let now $j$ be a fixed natural number $\geqq 1$ and $\leqq p$. By an isotopic deformation of each one of the pairs $G_{i}, H_{i}, i=1, \cdots$, $p$, on $N$, such that $q_{j}$ moves on $G_{j}$ and $q_{i}$ moves to $o_{j}$, we obtain a new fundamental system $A_{j i}, B_{j i}, i=1, \cdots, p$, on $N$ with base point $o_{j}$, such that the carrier of $A_{j j}$ is precisely $G_{j}$. Then

$$
\begin{aligned}
& \pi_{1}\left(N, o_{j}\right) \approx F_{j}=\left(a_{j 1}, b_{j 1}, \cdots, a_{j p}, b_{j p}: \prod_{i=1}^{p}\left[a_{j i}, b_{j i}\right]\right), \\
& j=1, \cdots, p \geqq 2 .
\end{aligned}
$$

Let $\bar{\Delta}_{j}$ be the smallest normal subgroup of $F_{j}$, containing the element $\left[a_{j j}, c_{j j}\right]$, where $c_{j j}$ is the element of $F_{j}$ corresponding to the loop obtained from the geodesic $G_{j}^{\prime}$. Let finally $\bar{D}_{j} \rightarrow N$ be the regular covering corresponding to $\bar{\Delta}_{j}$. We observe that the group of covering translations is $F_{j} / \bar{\Delta}_{j}$.

Under the above hypotheses, the modified conjectures are the following.

(1) For any $p \geqq 2$, there exists a $j(\geqq 1$ and $\leqq p)$, such that the group $F_{j} / \bar{\Delta}_{j}$ has no elements of finite order.

(2) For the same $j$ as above, $\bar{D}_{j}$ is planar.

The Conjectures (1) and (2) imply the Poincaré conjecture. The method is precisely that described in [2, Nos. 1-4], with the only difference that, at the end we "pass to the geodesics" $G_{i}, H_{i}$ and $G_{i}^{\prime}$ of $A_{i}, B_{i}$ and $A_{i}^{\prime}$ respectively, $i=1, \cdots, p$, see $[2$, p. 365, 11. 3-9 and 11. 8-6 from the bottom] and [3, Lemma (12.2)].

Let now $\Phi_{j}$ be the free group freely generated by $a_{j 1}, b_{j 1}, \cdots$, $a_{j p}, b_{j p}$. We observe that $c_{j j}=b_{j j} \tau_{j}$, where $\tau_{j} \in\left[\Phi_{j}, \Phi_{j}\right]$. Then

$$
\begin{aligned}
\bar{\Delta}_{j} & =\left\langle\left[a_{j j}, b_{j j} \tau_{j}\right]\right\rangle \\
F_{j} / \bar{\Delta}_{j} & \approx\left(a_{j 1}, b_{j 1}, \cdots, a_{j p}, b_{j p}: \prod_{i=1}^{p}\left[a_{j i}, b_{j i}\right],\left[a_{j j}, b_{j j} \tau_{j}\right]\right) .
\end{aligned}
$$

Therefore our Conjectures (1) and (2) above are essentially special cases of $[2$, p. 365, Conjectures (5.1) and (5.2)]. That the last group 
has no elements of finite order has been proved recently by Elvira S. Rapaport [5].

The Conjectures (1) and (2) avoid the obstacles raised by the examples of [1]. Actually, let us consider the first (or third) example of [1], we note that $p=2$. Let $G_{1}$ and $G_{1}^{\prime}$ be the closed geodesics on $N$ corresponding to the words $a_{1}$ and $b_{1}\left[b_{1}^{-1}, b_{2}\right]$ (or $a_{1}$ and $b_{1}\left[b_{2}, a_{2}\right]$ ) respectively. Then $G_{1}$ and $G_{1}^{\prime}$ are primary and simple [3, Lemma (12.2) ], and they have only one point $o_{1}$ in common. Thus there is a simple loop $L$ on $N$, which is homotopic on $N$ to

$$
L_{1} L_{1}^{\prime} L_{1}^{-1}\left(L_{1}^{\prime}\right)^{-1}
$$

where $L_{1}$ and $L_{1}^{\prime}$ are the loops with base point $o_{1}$, obtained from the geodesics $G_{1}$ and $G_{1}^{\prime}$ respectively. Therefore $F_{1} / \bar{\Delta}_{1}$ has no elements of finite order, and $\bar{D}_{1}$ is planar [3, Lemma (8.1)]. Hence the first and third examples of [1] are no counter examples to the above Conjectures (1) and (2). As far as the second example of [1] is concerned, this shows that it is indispensable that the geodesics $G_{i}^{\prime}, i=1, \cdots, p$, be simple, see $[2$, p. $365,11.8-6$ from the bottom $]$.

It was the first example of [1] which compelled me to modify the conjectures, from $[2$, p. 365 , Conjectures (5.1) and (5.2) $]$ to another form, where the property " $b_{j} \tau_{j}$ being cyclically reduced" comes into play. Finally, it was the third example of [1] which compelled me to modify the conjectures once again, and give them the above form (1) and (2).

Concluding the present note we would like to emphasize that, $\bar{\Delta}_{j}$ depends on the point $o_{j}, j=1, \cdots, p$. Thus the proper selection of the index $j$, and of the point $o_{j}$, may affect the validity of the Conjecture (2).

\section{BIBLIOGRAPHY}

1. Bernard Maskit, On a conjecture concerning planar coverings of surfaces, Bull. Amer. Math. Soc. 69 (1963), 396-398.

2. C. D. Papakyriakopoulos, A reduction of the Poincare conjecture to other conjectures, Bull. Amer. Math. Soc. 68 (1962), 360-366.

3. - A reduction of the Poincare conjecture to group theoretic conjectures, Ann. of Math. (2) 77 (1963), 250-305.

4. Bruce L. Reinhart, Algorithms for Jordan curves on compact surfaces, Ann. of Math. (2) 75 (1962), 209-222.

5. Elvira S. Rapaport, On a problem of Papakyriakopoulos, Bull. Amer. Math. Soc. 69 (1963), 402-404.

Princeton University 